\title{
Qualidade de sementes de tabaco durante o processo de pelotização e armazenamento
}

\author{
Quality of tobacco seeds during the pelletizing process and storage
}

\author{
Carla Massimo Caldeira ${ }^{\mathrm{I}}$ Maria Laene Moreira de Carvalho ${ }^{I I}$ \\ Renato Mendes Guimarães ${ }^{I I}$ Stefânia Vilas Boas Coelho ${ }^{\text {II }}$
}

\section{RESUMO}

O processo de pelotização pode afetar a qualidade das sementes por ocasião da semeadura, por isso, para avaliar o efeito desse processo na qualidade das sementes, foram utilizadas sementes de duas cultivares de tabaco 'CSC 467' e 'CSC 07', coletadas em diferentes etapas do processo de beneficiamento e pelotização: 1) sementes nuas não beneficiadas; 2) sementes nuas beneficiadas; 3) sementes pelotizadas; 4) sementes pelotizadas e coloridas. Para avaliação da qualidade das sementes nessas diferentes etapas, foram realizados os seguintes testes: germinação, primeira contagem de germinação, indice de velocidade de germinação, teste de emergência e índice de velocidade de emergência. Posteriormente, as sementes foram armazenadas por seis e doze meses e sua qualidade avaliada pelos mesmos testes e determinações. O processo de pelotização avaliado não influencia na germinação final de sementes de tabaco, mas atrasa o processo germinativo. As sementes de tabaco das cultivares 'CSC 467' e 'CSC 07' mantêm sua qualidade durante os 12 meses de armazenamento em câmara fria, com temperatura de $10^{\circ} \mathrm{C}$ e umidade relativa de $50 \%$.

Palavras-chave: Nicotiana tabacum L., germinação, emergência.

\section{ABSTRACT}

The pelletizing process may affect the quality of seeds during sowing. In order to evaluate the effect of this process on seed quality it was used seeds from three tobacco cultivars, 'CSC 467' and 'CSC 07' collected in different processing and pelletizing process stages: 1) non processed nude seeds; 2) processed nude seeds; 3) pelletized seeds; 4) pelletized and colored seeds. For the evaluation of seed quality in these different stages it was performed the following tests: Germination, First Count of Germination, Germination Speed Index, Initial and Final Stand (Emergence) and Emergence
Speed Index. Posteriorly, seeds were stored for six and twelve months and their quality was evaluated by the same tests and determinations. The evaluated pelletizing process does not influence final germination of the tobacco seeds, but delays the germination process. Tobacco seeds from cultivars 'CSC 467 ' and 'CSC 07 ' maintain their quality during the 12 months of storage in cold chamber with temperature of $10^{\circ} \mathrm{C}$ and relative humidity of $50 \%$.

Key words: Nicotiana tabacum L., germination, emergence.

\section{INTRODUÇÃO}

O tabaco é, atualmente, a mais importante cultura agrícola não alimentícia do planeta e contribui substancialmente para as economias de mais de 150 países, sendo o Brasil, na atualidade, o segundo maior produtor do mundo e o principal exportador.

Um dos entraves encontrados no cultivo do tabaco está relacionado ao tamanho, forma e peso das sementes, o que dificulta a semeadura, porque cada grama de sementes contém cerca de 16 mil unidades (BRASIL, 2009). Por esse motivo, as sementes comerciais são pelotizadas para aumentar o tamanho e facilitar a semeadura, sendo que a pelotização consiste no revestimento das sementes com um material seco, inerte, de granulometria fina e um material cimentante (adesivo). Esse tratamento confere à semente uma forma arredondada, aumentando o seu tamanho,

\footnotetext{
IDepartamento de Agricultura, Universidade Federal de Lavras (UFLA), 37200-000, Lavras, MG, Brasil. E-mail: carlaufla@yahoo.com.br. Autor para correspondência.

IIUniversidade Federal de Lavras (UFLA), Lavras, MG, Brasil. 
facilitando assim a sua distribuição, seja manual ou mecânica (GRELLIER et al., 1999).

O uso de sementes pelotizadas pode ter alguns problemas, pois a pelota formada ao redor da semente pode afetar seu desempenho durante a germinação (CARVALHO \& NOVEMBRE, 2011). Isso devido ao arranjo das partículas finas da pelota o que dificulta a emissão da raiz primária e a troca de gases, causando geralmente atraso no processo de germinação (SILVA et al., 2002; NASCIMENTO et al., 2009).

Outros trabalhos demonstraram que sementes nuas de várias espécies germinam mais rápido do que as sementes pelotizadas (SAMPAIO \& SAMPAIO, 1994; FRANZIN et al., 2004; PIRES et al., 2004). Além disso, as sementes de tabaco são fotoblásticas positivas (LEUBNERMETZGER \& MEINS, 2000), de forma que o revestimento pode induzir a uma fotoinibição, afetando negativamente a germinação das sementes, portanto, a pelota utilizada no revestimento das sementes deve se desintegrar ou abrir com facilidade, para não constituir resistência à germinação (BONOME, 2003).

Nesse sentido, é de extrema importância o conhecimento da qualidade das sementes durante todas as etapas do processo de pelotização, bem como o efeito da pelota na geminação, visando à obtenção de sementes de qualidade, com germinação rápida e uniforme. Objetivou-se avaliar a qualidade de sementes de tabaco de duas cultivares, durante o processo de pelotização ao longo do armazenamento.

\section{MATERIAL E MÉTODOS}

O experimento foi conduzido no Laboratório Central de Análise de Sementes do Departamento de Agricultura da Universidade Federal de Lavras (UFLA) e na empresa Souza Cruz em Rio Negro - PR, sendo a pelotização realizada na empresa e as avaliações realizadas no laboratório.

As sementes utilizadas foram de duas cultivares de tabaco ('CSC 467' e 'CSC 07'), colhidas em Rio Negro - PR, na safra de 2012, as quais foram coletadas em diferentes etapas do processo de beneficiamento e pelotização. Dessa forma, estabeleceram-se os seguintes tratamentos: 1) sementes nuas não beneficiadas; 2) sementes nuas beneficiadas; 3) sementes pelotizadas; 4) sementes pelotizadas e coloridas.

As sementes nuas não beneficiadas foram obtidas após trilhagem mecânica dos frutos secos e pré-limpeza em peneiras para eliminação das impurezas mais grosseiras e para obtenção das sementes nuas beneficiadas, o beneficiamento foi realizado em equipamento de separação por densidade da marca Seed Tech System, modelo STS-MC3 - Mobile Cabinet Separator. A pelotização foi efetuada de acordo com o padrão estabelecido pela empresa produtora e, ao final do processo, as sementes foram secas a $40^{\circ} \mathrm{C}$, por aproximadamente 45 minutos, até atingirem $1,5 \%$ de umidade.

As avaliações dos tratamentos foram efetuadas logo após o processo de pelotização das sementes e após 6 e 12 meses de armazenamento em câmara fria (temperatura $10^{\circ} \mathrm{C}$ e umidade relativa de 50\%) com as sementes armazenadas em embalagens aluminizadas. Para avaliação da qualidade das sementes, foram realizados os seguintes testes: germinação, primeira contagem de germinação, índice de velocidade de germinação, teste de emergência e índice de velocidade de emergência.

No teste de germinação, quatro repetições de 50 sementes foram semeadas em caixas de acrílico com tampa $(11 \times 11 \times 3,5)$, tipo gerbox, sobre substrato papel mata-borrão, umedecido com solução de $\mathrm{KNO}_{3}(0,2 \%)$ em quantidade equivalente a 2,5 vezes o peso do substrato seco. As sementes foram mantidas em BOD (Biological Oxygen Demand) com temperatura alternada de 20$30^{\circ} \mathrm{C}$ e fotoperíodo de oito horas e intensidade de luz acima de 2.000lux (BRASIL, 2009). O número de sementes germinadas foi avaliado diariamente, com o auxílio de lupa, para obtenção do índice de velocidade de germinação (MAGUIRE, 1962). Os resultados de germinação foram expressos em porcentagem de plântulas normais com avaliação aos sete dias após a montagem do teste, para obtenção da primeira contagem de germinação, e aos 16 dias (BRASIL, 2009).

$O$ teste de emergência foi conduzido em sistema float, sendo a semeadura realizada em substrato de fibra de coco, previamente umedecido (aproximadamente 1 litro de água $\mathrm{kg}^{-1}$ de substrato), colocado em bandejas de isopor com 200 células. Após semeadura, as bandejas foram colocadas no sistema float, que se caracteriza por manter as bandejas de isopor perfuradas, flutuando sobre uma lâmina de água de aproximadamente três centímetros e mantidas em casa de vegetação com temperatura média de $35^{\circ} \mathrm{C}$. A avaliação do número de plântulas emergidas e com o primeiro par de folhas foi realizada diariamente, para a obtenção do índice de velocidade de emergência (MAGUIRE, 1962), no décimo quinto e vigésimo primeiro dia, 
para a obtenção dos dados de primeira contagem e porcentagem de emergência.

O delineamento utilizado foi inteiramente ao acaso e os dados foram interpretados estatisticamente por meio de análise de variância em esquema fatorial $4 \times 3$, sendo 4 etapas de pelotização (1-sementes nuas não beneficiadas, 2-sementes nuas beneficiadas, 3-sementes pelotizadas, 4-sementes pelotizadas e coloridas) e 3 épocas de armazenamento (0, 6 e 12 meses). Para as diferentes, foram realizadas três análises, separadamente. As médias foram comparadas pelo teste de Scott-Knott, ao nível de $5 \%$ de probabilidade.

\section{RESULTADOS E DISCUSSÃO}

Para a germinação e vigor avaliado pelos testes de índice de velocidade, primeira contagem de germinação e germinação, houve interação significativa entre as etapas do processo de pelotização e tempo de armazenamento, exceto no teste de germinação da cultivar 'CSC 467', para a qual apenas os dados do processo de pelotização foram significativos.

Por meio dos resultados de primeira contagem de germinação, observa-se que a pelota atrasou o processo de germinação das sementes, embora não tenham afetado negativamente a germinação (Tabela 1). Esses resultados estão de acordo com PIRES et al. (2004) e SILVA et al. (2002), os quais também verificaram que a germinação não foi reduzida pela pelotização, apesar da velocidade de germinação das sementes ter sido afetada, devido à presença de uma barreira física. Segundo COSTA et al. (2001), as sementes recobertas demoram mais tempo para absorver a umidade, podendo retardar a germinação em até 48 horas, quando comparada às sementes nuas.

No índice de velocidade de germinação, primeira contagem de germinação, e germinação, independente da época de avaliação, as sementes nuas beneficiadas foram mais vigorosas, quando comparadas às nuas sem beneficiamento (Tabela 1). Esses resultados eram esperados, uma vez que o beneficiamento elimina os materiais indesejáveis e sementes mal formadas ou mais leves, que acompanham as sementes e melhoram a pureza física dos lotes e, consequentemente, o seu vigor (FRANZIN \& MENEZES, 2002).

Nas sementes nuas beneficiadas (tratamento 2), os valores de velocidade de germinação e primeira contagem de germinação não foram afetados negativamente durante os meses de armazenamento (Tabela 1). Para as sementes pelotizadas, houve uma redução na velocidade de germinação aos seis meses de armazenamento, com posterior elevação dos índices aos 12 meses. Tais resultados concordam com OLIVEIRA et al. (2003) que relataram que o material de revestimento pode dificultar a entrada de oxigênio durante o processo de germinação e, no estágio mais avançado do

Tabela 1 - Valores médios de índice de velocidade de germinação - (IVG), primeira contagem de germinação - (PCG\%) e germinação $(\mathrm{G} \%)$ de sementes de tabaco das cultivares ' $\mathrm{CSC} 467$ ' e 'CSC 07', armazenadas e submetidas aos diferentes tratamentos.

\begin{tabular}{|c|c|c|c|c|c|c|c|c|c|}
\hline \multirow[t]{2}{*}{ Tratamentos } & \multicolumn{3}{|c|}{ 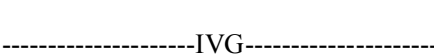 } & ---------. & --PCG- & ----------. & \multirow{2}{*}{\multicolumn{2}{|c|}{ G }} & \\
\hline & 0 & 6 & 12 & 0 & 6 & 12 & & & \\
\hline 1 - nuas sem benef. & $7,00 \mathrm{Ab}$ & $5,10 \mathrm{Bb}$ & $8,37 \mathrm{Ac}$ & $61 \mathrm{Ab}$ & $47 \mathrm{Bb}$ & $63 \mathrm{Ac}$ & & $66 \mathrm{~b}$ & \\
\hline 2 - nuas benef. & $10,08 \mathrm{Aa}$ & $9,28 \mathrm{Aa}$ & $10,39 \mathrm{Aa}$ & $79 \mathrm{Aa}$ & $78 \mathrm{Aa}$ & $80 \mathrm{Aa}$ & & $83 \mathrm{a}$ & \\
\hline 3 - pelotizadas & $6,12 \mathrm{Ab}$ & $3,25 \mathrm{Bc}$ & 7,49 Ac & $3 \mathrm{Bc}$ & $0 \mathrm{Bc}$ & $61 \mathrm{Ac}$ & & $83 \mathrm{a}$ & \\
\hline 4 - pelot. e coloridas & $4,69 \mathrm{Bc}$ & $2,90 \mathrm{Cc}$ & $8,36 \mathrm{Ac}$ & $7 \mathrm{Bc}$ & $0 \mathrm{Bc}$ & $72 \mathrm{Ab}$ & & $81 \mathrm{a}$ & \\
\hline $\mathrm{CV}(\%)$ & 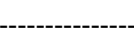 & $-15,08$ & 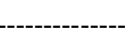 & ------- & $-13,38$ & --------- & & 8,36 & \\
\hline \multirow[t]{2}{*}{ Tratamentos } & (- & $----I V G-$ & ---------- & $------\cdot$ & --PCG- & - & & --G---- & \\
\hline & 0 & 6 & 12 & 0 & 6 & 12 & 0 & 6 & 12 \\
\hline 1 - nuas sem benef. & $9,09 \mathrm{Ab}$ & $5,96 \mathrm{Cb}$ & $7,78 \mathrm{Bc}$ & $71 \mathrm{Ab}$ & $51 \mathrm{Bb}$ & $55 \mathrm{Bb}$ & $74 \mathrm{Ab}$ & $54 \mathrm{Bb}$ & $62 \mathrm{Bb}$ \\
\hline 2 - nuas benef. & $11,24 \mathrm{Aa}$ & $9,77 \mathrm{Ba}$ & $10,94 \mathrm{Aa}$ & $86 \mathrm{Aa}$ & $85 \mathrm{Aa}$ & $81 \mathrm{Aa}$ & $88 \mathrm{Aa}$ & $89 \mathrm{Aa}$ & $87 \mathrm{Aa}$ \\
\hline 3 - pelotizadas & $5,51 \mathrm{Ac}$ & $3,38 \mathrm{Bc}$ & $5,82 \mathrm{Ad}$ & $5 \mathrm{Bc}$ & $0 \mathrm{Bc}$ & $23 \mathrm{Ac}$ & $79 \mathrm{Ab}$ & $78 \mathrm{Aa}$ & $85 \mathrm{Aa}$ \\
\hline 4 - pelot. e coloridas & 3,31 Bd & $3,58 \mathrm{Bc}$ & $9,10 \mathrm{Ab}$ & $5 \mathrm{Bc}$ & $0 \mathrm{Bc}$ & $85 \mathrm{Aa}$ & $80 \mathrm{Bb}$ & $81 \mathrm{Ba}$ & $91 \mathrm{Aa}$ \\
\hline
\end{tabular}

Médias seguidas da mesma letra, minúscula na coluna e maiúscula na linha, pertencem ao mesmo grupo pelo teste de Scott-Knott, a 5\% de probabilidade. 
armazenamento, essa impermeabilização pode ter sido diminuída.

Além disso, o armazenamento das sementes pelotizadas à baixa temperatura e no escuro pode ter aumentado a síntese de giberelinas (RESENDE et al., 2009), o que também está de acordo com PEREIRA \& MAEDA (1986), que verificaram possível relação entre o tratamento de frio e aumento nos níveis endógenos do hormônio. Como em espécies fotoblásticas positivas, a giberelina pode substituir a luz na germinação das sementes, o aumento do seu nível durante o armazenamento pode ter favorecido a germinação.

Para os dados de índice de velocidade de emergência, primeira contagem e porcentagem de emergência, houve interação significativa entre as etapas do processo de pelotização e tempo de armazenamento para a cultivar ' $\mathrm{CSC}$ 07', enquanto, para a cultivar ' CSC 467', somente o processo de pelotização foi significativo. Nas sementes submetidas ao processo de pelotização (tratamento 4), não houve diferenças de vigor em relação às sementes nuas beneficiadas (tratamento 2), indicando que a pelota não influenciou a emergência das plântulas no campo (Tabela 2). No entanto, observa-se uma menor velocidade de emergência para as sementes pelotizadas, de forma que a pelota afetou negativamente a velocidade de emergência das sementes, sem contudo afetar no estante final. Esse resultado corrobora os obtidos para outras espécies, como tomate (OLIVEIRA et al., 2003), brócolis (COSTA \& TARANTO, 2005) e braquiária (SANTOS et al., 2010).

Para a cultivar 'CSC 07', observa-se, pelos resultados da porcentagem de emergência (Tabela 2), que o vigor das sementes permaneceu inalterado ao longo do armazenamento, exceto para as sementes nuas sem beneficiamento (tratamento 1). As sementes deterioradas tendem a perder a qualidade mais rápido durante o armazenamento (MARCOS FILHO,1999).

Fica evidente que a pelota não afeta a germinação final, somente atrasa o processo germinativo, provavelmente, devido ao impedimento físico provocado. No entanto, estudos adicionais devem ser realizados para adequar os materiais utilizados no processo de pelotização, de modo que esses não afetem a velocidade de germinação.

\section{CONCLUSÃO}

O processo de pelotização avaliado não afeta a germinação, apenas atrasa o processo germinativo das sementes. Independente da cultivar, ocorre restrição ao processo germinativo das sementes pelotizadas aos 6 meses de armazenamento, minimizadas aos 12 meses. As sementes de tabaco das cultivares 'CSC 467' e 'CSC 07' mantêm sua qualidade fisiológica durante os 12 meses de armazenamento em câmara fria.

Tabela 2 - Valores médios de índice de velocidade de emergência - (IVE), primeira contagem - (PC\%) e porcentagem de emergência (PE\%) de plântulas de tabaco oriundas de sementes das cultivares 'CSC 467' e 'CSC 07', armazenadas e submetidas aos diferentes tratamentos.

\begin{tabular}{|c|c|c|c|c|c|c|c|c|c|}
\hline \multirow{2}{*}{ Tratamentos } & \multirow{2}{*}{\multicolumn{3}{|c|}{ IVE }} & \multirow{2}{*}{\multicolumn{3}{|c|}{$\mathrm{PC}$}} & \multirow{2}{*}{\multicolumn{3}{|c|}{$\mathrm{PE}$}} \\
\hline & & & & & & & & & \\
\hline 1 - nuas sem benef. & \multicolumn{3}{|c|}{$2,17 \mathrm{c}$} & \multicolumn{3}{|c|}{$65 \mathrm{~b}$} & \multicolumn{3}{|c|}{$66 \mathrm{~b}$} \\
\hline 2 - nuas benef. & \multicolumn{3}{|c|}{$2,78 \mathrm{a}$} & \multicolumn{3}{|c|}{$85 \mathrm{a}$} & \multicolumn{3}{|c|}{$88 \mathrm{a}$} \\
\hline 3 - pelotizadas & \multicolumn{3}{|c|}{$2,50 \mathrm{~b}$} & \multicolumn{3}{|c|}{$81 \mathrm{a}$} & \multicolumn{3}{|c|}{$85 \mathrm{a}$} \\
\hline 4 - pelot. e coloridas & \multicolumn{3}{|c|}{$2,52 \mathrm{~b}$} & \multicolumn{3}{|c|}{$81 \mathrm{a}$} & \multicolumn{3}{|c|}{$83 \mathrm{a}$} \\
\hline $\mathrm{CV}(\%)$ & \multicolumn{3}{|c|}{12,81} & \multicolumn{3}{|c|}{10,93} & \multicolumn{3}{|c|}{10,26} \\
\hline \multirow[t]{2}{*}{ Tratamentos } & - & ---IVE-- & ------- & - & ---PC--- & - & ------ & ---PE--- & \\
\hline & 0 & 6 & 12 & 0 & 6 & 12 & 0 & 6 & 12 \\
\hline 1 - nuas sem benef. & $2,08 \mathrm{Ab}$ & $2,29 \mathrm{~b}$ & $1,82 \mathrm{Ab}$ & $76 \mathrm{Ab}$ & $74 \mathrm{Ab}$ & $54 \mathrm{Bc}$ & $79 \mathrm{Aa}$ & $74 \mathrm{Ab}$ & $54 \mathrm{Bb}$ \\
\hline 2 - nuas benef. & $3,16 \mathrm{Aa}$ & $2,79 \mathrm{Aa}$ & $3,09 \mathrm{Aa}$ & 89 Аа & $87 \mathrm{Aa}$ & $90 \mathrm{Aa}$ & 89 Аа & $87 \mathrm{Aa}$ & $91 \mathrm{Aa}$ \\
\hline 3 - pelotizadas & $3,22 \mathrm{Aa}$ & $2,09 \mathrm{Bb}$ & $2,10 \mathrm{Bb}$ & $85 \mathrm{Aa}$ & $81 \mathrm{Aa}$ & $86 \mathrm{Aa}$ & $85 \mathrm{Aa}$ & $82 \mathrm{Aa}$ & $86 \mathrm{Aa}$ \\
\hline 4 - pelot. e coloridas & 3,31 Aa & $2,10 \mathrm{Ba}$ & $1,88 \mathrm{Bb}$ & $88 \mathrm{Aa}$ & $86 \mathrm{Aa}$ & $73 \mathrm{Bb}$ & $88 \mathrm{Aa}$ & $88 \mathrm{Aa}$ & $80 \mathrm{Aa}$ \\
\hline
\end{tabular}

Médias seguidas da mesma letra, minúscula na coluna e maiúscula na linha, pertencem ao mesmo grupo pelo teste de Scott-Knott, a $5 \%$ de probabilidade. 


\section{REFERÊNCIAS}

BONOME, L.T.S. Condicionamento fisiológico e revestimento de sementes de Brachiaria brizantha cultivar 'Marandu'. 2003. 99f. Dissertação (Mestrado em Fitotecnia) - Universidade Federal de Lavras, Lavras, MG.

BRASIL. Ministério da Agricultura, Pecuária e Abastecimento. Regras para análise de sementes. Brasília: Secretaria de Defesa Agropecuária, SNDA/DNDV/CLAV, 2009. 395p.

CARVALHO, C.; NOVEMBRE, A.L.C. Avaliação da qualidade de sementes de fumo, nuas e revestidas, pelo teste de condutividade elétrica. Revista Brasileira de Sementes, Londrina, v.33, n.1, p.177-185, 2011. Disponível em: <http://www.scielo.br/scielo. php? script $=$ sci arttext\&pid $=$ S0101-31222011000100020>. Acesso em: 20 jan. 2014. doi: 10.1590/S0101-31222011000100020.

COSTA, C.E.L. et al. Sementes de cenoura, Daucus carota L., revestidas e peliculadas: germinação e vigor durante o armazenamento. Revista Brasileira de Armazenamento, Viçosa, v.26, p.36-45, 2001.

COSTA, M.A.; TARANTO, O.P. The effect of initial size on the germination of pelleted broccoli seeds. Transactions of the ASAE, Saint Joseph, v.48, n.5, p.1677-1680, 2005.

FRANZIN, S.M. et al. Avaliação do vigor de sementes de alface nuas e peletizadas. Revista Bras ileira de Sementes, Pelotas, v.26, n.2, p.114-118, 2004. Disponível em: <http://www.scielo.br/ scielo.php?script=sci_arttext\&pid=S0101-31222004000200016>. Acesso em: 05 fev. 2014. doi: 10.1590/S0101-31222004000200016.

FRANZIN, S.M.; MENEZES, N.L. Análise de sementes: 2., temperaturas e qualidade de água para germinação de sementes peletizadas de alface. Santa Maria: UFSM, 2002. 4p. (Informe Técnico, 1).

GRELLIER, P. et al. Transfer and water-retention properties of seed-pelleting materials. European Journal of Agronomy, London, v.10, n.1, p.57-65, 1999. Disponível em: <http://www. sciencedirect.com/science/article/pii/S1161030198000501>. Acesso em: 30 mar. 2014. doi: 10.1016/S1161-0301(98)00050-1.

LEUBNER-METZGER, G.; MEINS, F.J.R. Sense transformation reveals a novel role for class I b-1,3-glucanase in tobacco seed germination. Plant Journal, Oxford, v.23, n.2, p.215-221, 2000. Disponível em: <http://onlinelibrary.wiley.com/enhanced/ doi/10.1046/j.1365-313x.2000.00773.x/>. Acesso em: 07 mar. 2014. doi: 10.1046/j.1365-313x.2000.00773.x.

MAGUIRE, J.D. Speeds of germination aid selection and evaluation for seedling emergence and vigor. Crop Science, Madison, v.2, n.2, p.176-177, 1962.
MARCOS FILHO, J. Testes de vigor: importância e utilização. In: KRZYZANOWSKI, F.C. et al. (Ed.). Vigor de sementes: conceitos e testes. Londrina: ABRATES, 1999. Cap.1, p.1-21.

NASCIMENTO, W.M. et al. Germinação de sementes de cenoura osmoticamente condicionadas e peletizadas com diversos ingredientes. Horticultura Brasileira, Brasília, v.27, n.1, p.12-16, 2009. Disponível em: $<\mathrm{http}: / /$ www.scielo.br/scielo.php?script=sci arttext\&pid=S0102-05362009000100003 $>$. Acesso em: 20 mar. 2014. doi: 10.1590/S0102-05362009000100003.

OLIVEIRA, J.A. et al. Efeito de diferentes materiais de peletização na deterioração de sementes de tomate durante o armazenamento. Revista Brasileira de Sementes, Pelotas, v.25, n.2, p.20-27, 2003. Disponível em: <http://www.scielo. $\mathrm{br} / \mathrm{scielo}$.php? pid $=\mathrm{S} 0101-31222003000400004 \& \mathrm{script}=\mathrm{sci}$ arttext>. Acesso em: 04 abr. 2014. doi: 10.1590/S010131222003000400004 .

PEREIRA, M.F.D.A.; MAEDA, J.A. Environmental and endogenous control of germination of Vitis vinifera seeds. Seed Science and Technology, Zürich, v.14, p.227-235, 1986.

PIRES, L.L. et al. Armazenamento de sementes de feijão revestidas com polímeros e tratadas com fungicidas. Pesquisa Agropecuária Brasileira, Brasília, v.39, n.7, p.709-715, 2004. Disponível em: <http://www.scielo.br/scielo.php?pid=S0100204X2004000700013\&script=sci_arttext $>$. Acesso em: 10 fev. 2014. doi: 10.1590/S0100-204X2004000700013.

RESENDE, M.L. et al. Influência da luz e giberelina na velocidade de germinação das sementes de cafeeiro (Coffea arabica L.). Coffee Science, Lavras, v.4, n.2, p.149-154, 2009. Disponível em: <http://www.coffeescience.ufla.br/index.php/Coffeescience/ article/view/179>. Acesso em: 22 jan. 2014.

SAMPAIO, T.G.; SAMPAIO, N.V. Recobrimento de sementes. Informativo ABRATES, Brasília, v.4, n.3, p.20-52, 1994.

SANTOS, F.C. et al. Tratamento químico, revestimento e armazenamento de sementes de Brachiaria brizantha cv. 'Marandu'. Revista Brasileira de Sementes, Londrina, v.32, n.3, p.69-78, 2010. Disponível em: <http:/www.scielo.br/scielo. php? script $=$ sci_arttext\&pid $=$ S0101-31222010000300008>. Acesso em: 03 abr. 2014. doi: 10.1590/S0101-31222010000300008.

SILVA, J.B.C. et al. Desempenho de sementes pelotizadas de alface em função do material cimentante e da temperatura de secagem dos péletes. Horticultura Brasileira, Brasília, v.20, n.1, p.67-70, 2002. Disponível em: <http://www.scielo.br/scielo.php?pid=S010205362002000100013\&script $=$ sci_arttext $>$. Acesso em: 01 abr. 2014. doi: 10.1590/S0102-05362002000100013. 